\title{
\begin{tabular}{l|l} 
POLITIQUES \& & Politiques et management public
\end{tabular}

\section{Le revenu de solidarité active : changements et continuités institutionnelles en phase d'expérimentation}

Social income policy : institutional change and continuity in its trial phase

Patricia Loncle, Virginie Muniglia et Thierry Rivard

\section{(2) OpenEdition \\ Journals}

Édition électronique

URL : http://journals.openedition.org/pmp/2189

DOI : 10.4000/pmp.2189

ISSN : 2119-4831

Éditeur

Institut de Management Public (IDPM)

Édition imprimée

Date de publication : 15 mars 2010

Pagination : 35-54

ISSN : 0758-1726

\section{Référence électronique}

Patricia Loncle, Virginie Muniglia et Thierry Rivard, «Le revenu de solidarité active : changements et continuités institutionnelles en phase d'expérimentation », Politiques et management public [En ligne], Vol 27/2 | 2010, mis en ligne le 15 mars 2012, consulté le 07 mai 2019. URL : http:// journals.openedition.org/pmp/2189; DOI : 10.4000/pmp.2189 


\title{
LE REVENU DE SOLIDARITÉ ACTIVE : CHANGEMENTS ET CONTINUITÉS INSTITUTIONNELLES EN PHASE D'EXPÉRIMENTATION
}

\author{
Patricia Loncle ${ }^{1}$ \\ Virginie Muniglia ${ }^{1}$ \\ Thierry Rivard ${ }^{2}$
}

\begin{abstract}
Résumé
L'analyse de la mise en œuvre de l'expérimentation du Revenu de solidarité active (RSA) dans cinq départements français, permet de s'interroger sur la nature et l'ampleur des changements effectivement introduits lors de cette phase précédant la généralisation. Concernant la portée des décisions départementales, le changement apparaît ainsi contraint par les héritages du RMI, d'une part, et par l'intégration forte dans le jeu institutionnel de l'Agence nationale pour les solidarités actives, instance régulatrice et pourvoyeuse d'expertise, d'autre part. Du point de vue de la mise en œuvre, on constate l'introduction d'innovations institutionnelles dont la portée reste cependant très dépendante des configurations territoriales. Ainsi, si le changement paraît indéniable du point de vue des instruments et des acteurs mobilisés, il reste plus limité si l'on considère le cadre d'interaction du RSA et ses orientations politiques.
\end{abstract}

Mots clefs

changement, expérimentation, configurations locales d'acteurs, décision, mise en œuvre.

Abstract The analysis of the experimentation of the Revenu de solidarité active $(R S A)$ in five French Départements allows a questioning about the nature and the scope of the changes introduced by this phase which precede the generalisation. Regarding the impact of decision, the change appears as compelled, firstly, by the legacy of the RMI and, secondly, by the strong integration in the institutional game of the Agence nationale pour les solidarités actives - a regulating and expert authority. Regarding the implementation, it can be noted that, if institutional innovations were introduced, they remain limited if one considers the interaction frame of the RSA and its political orientations.

Key words change, experimentation, local configurations of actors, decision, implementation.

\footnotetext{
${ }^{1}$ École des Hautes Études en Santé Publique (EHESP) de Rennes

${ }^{2}$ Laboratoire étude recherche et formation en action sociale (LERFAS) de Tours
}

Revue POLITIQUES ET MANAGEMENT PUBLIC, Volume 27, n² 2, 2010.

(C) Institut de Management Public - 2010. 
Introduction Dans le cadre de l'expérimentation du RSA, la stratégie de l'État, a consisté à fixer un cadre d'action général, tout en laissant aux Départements un certain nombre de choix : la délimitation du territoire d'expérimentation, le montant du barème et le calcul de la prestation, la nature des moyens humains mobilisés, la détermination partielle du public pris en considération... En fonction des situations économiques, des orientations politiques, de la stratégie antérieure en matière d'insertion, des constats opérés localement, certains Départements poursuivent des objectifs propres en complément de ceux affichés pour l'expérimentation.

Les éléments de contexte de l'expérimentation sont les suivants. Un mouvement de décentralisation des compétences de l'État social vers les Départements, considérablement accentué au cours de la dernière décennie. Co-gestionnaires d'un certain nombre de fonds sociaux et de minima sociaux depuis la première vague de décentralisation de 19821983, la loi du 18 décembre 2003 les a consacrés responsables du suivi et de la gestion de l'allocation du Revenu minimum d'insertion (RMI) et plus largement de la politique départementale d'insertion, celle du 13 août 2004 leur a confié la responsabilité des dossiers Fonds d'aide aux jeunes, Fonds de solidarité logement, personnes âgées, personnes handicapées, faisant de ces territoires les pivots de la politique sociale de notre pays (Le Lidec, 2008).

Ce mouvement comprend des formes de déconcentration déguisées, dénoncées par les Départements car les privant pour partie de leur marge de manœuvre (Warin et Avenel, 2007) et creuse des inégalités territoriales d'accès aux droits déjà importantes avant le mouvement de décentralisation (Destremeau et Messu, 2008). Cependant, face à ces contraintes communes, les Départements n'ont pas œuvré de la même manière (Le Bihan, Martin, Rivard, 2006 ; Loncle, Muniglia, Rivard et Rothé, 2008). Ainsi, les capacités des Départements à s'approprier ces dispositifs apparaissent contrastées, de même que leurs manières d'envisager et de définir les modalités d'accès des bénéficiaires aux différents types d'aide (Thierry, 2008).

À notre sens, ces particularités sont largement à rattacher à l'existence de configurations locales d'acteurs ${ }^{3}$ qui expliquent les spécificités territoriales observées d'un département à l'autre (Grémion, 1976 ; Lascoumes et Le Bourhis, 1998 ; Borraz, 1998 ; Borraz et Loncle, 2000 ; Pasquier, 2004 ; Négrier 2005).

\footnotetext{
${ }^{3}$ «Les configurations [territoriales] dans lesquelles les acteurs se meuvent sont donc la résultante d'histoires, de processus amorcés précédemment, qui sont autant de points d'appui sur des stratégies d'opportunité. La distribution spatiale et démographique, la stabilité politique du leader ou de la coalition dominante, l'existence ou non de contrepoids institutionnels et politiques (notamment les Conseils généraux), le degré d'apprentissage intercommunal préalable, les "traditions" d'exercice du pouvoir, sont des éléments de telles configurations. C'est à partir d'eux que se concrétise de façon différenciée, une dialectique entre coopération et conflit, notamment à l'occasion de projets de recomposition qui soulèvent, toujours des tensions existentielles ». (Négrier, 2005, p. 209)
} 
Une attention particulière est portée ici aux types d'acteurs impliqués, à leur perception de l'expérimentation, aux aspects diachroniques, géographiques et politiques qui structurent les territoires et à l'empreinte particulière de ces différents aspects sur les profils des actions publiques locales du point de vue de leur envergure, de leur caractère plus ou moins novateur et des valeurs qu'elles véhiculent.

L'influence des configurations locales d'acteurs apparaît d'autant plus significative que le dispositif examiné est expérimental, ce qui a priori laisse une marge d'appréciation non négligeable aux Départements. En ce sens, on se trouve dans une sorte de tradition inaugurée par les expérimentations précédant la généralisation du RMI (Cytermannn et Dindar, 2008) qui avaient permis aux collectivités locales de tester des mesures, certes, mais aussi de faire montre de leurs capacités d'initiative et de réactivité face aux questions d'insertion et de pauvreté.

Notre propos est par conséquent de montrer dans quelle mesure, en tenant compte de ces éléments de contexte, l'expérimentation du RSA permet d'apporter des changements dans l'accompagnement des bénéficiaires vis-à-vis des actions départementales déjà développées dans le cadre du RMI. Cette démarche renseigne non seulement sur la généralisation en cours mais également plus largement sur l'évolution des politiques sociales françaises. Pour travailler cette question, deux types de résultats d'analyse sont mobilisés : la décision politique et son impact relatif sur l'expérimentation ; les contraintes liées à la mise en œuvre de ce dispositif.

Le présent article est fondé sur l'analyse de cinq monographies de départements expérimentateurs du Revenu de Solidarité Active, sélectionnés par le comité de pilotage "enquêtes qualitatives » du Haut commissariat aux solidarités actives : la Côte-d'Or, I'Eure, la LoireAtlantique, le Nord et le Val-d'Oise.

\section{Encadré 1 - Présentation de l'enquête de terrain}

Une première vague d'enquête a été effectuée début 2008. Les investigations visaient à saisir les caractéristiques de la mise en œuvre du RSA : les orientations en matière d'insertion, l'antériorité des actions dans le cadre du RMI, l'état des relations entre les acteurs, l'organisation des moyens... les acteurs institutionnels les plus investis dans la mise en $œ u v r e$ du dispositif étaient concernés (Conseil général, Préfecture, Caisse d'allocations familiales).

Une deuxième vague d'enquête a été menée à l'automne 2008. Cette étape a été l'occasion d'élargir le cercle d'acteurs consultés. Les entretiens ont concerné l'Agence nouvelle des solidarités actives (ANSA), la Mutualité sociale agricole (MSA), les Maisons de l'emploi, les Directions départementales du travail de l'emploi et de la formation professionnelle (DDTEFP), les Plans locaux pour l'insertion et l'emploi (PLIE), les entreprises d'insertion, les centres de formation, les employeurs impliqués dans le dispositif. L'objectif était d'analyser l'implication des différents protagonistes, leurs avis sur le RSA et les évolutions introduites par l'expérimentation. 
La troisième vague d'enquête s'est déroulée en mars 2009. Elle a consisté en une série d'entretiens auprès des participants aux groupes témoins en Côte d'Or, en Loire-Atlantique et dans le Val d'Oise. L'objectif de ces entretiens était de recueillir le point de vue des participants aux groupes témoin sur le dispositif expérimental et sur leur expérience dans le cadre de cette initiative.

Pour les trois vagues, une liste d'acteurs a été arrêtée et soumise pour avis aux membres du comité de pilotage suscité, constitué de représentants de Conseils généraux et d'administrations et agences centrales -DREES, DARES, ANSA, CNAF...-. En outre, le recueil de données a été opéré à l'aide d'un guide d'entretien commun aux équipes et validé par le même comité de pilotage. Sur le terrain, les chercheurs ont veillé adapter les guides afin de réaliser des entretiens semi-directifs adaptés au questionnement.

L'équipe était composée de : Michèle Kergoat (Université de Rennes 1) Eric Legrand, Patricia Loncle, Hervé Michel, Virginie Muniglia (EHESP), Thierry Rivard et Suzanne Rosenberg (LERFAS).

Au total, cent quatre-vingt-quatre acteurs de l'expérimentation ont été interviewés, dont certains à plusieurs reprises. Vingt-et-un participants aux groupes témoins ont également été interrogés.

Deux points sont abordés tour à tour : la portée relative des décisions départementales quand les héritages liés à la décentralisation du RMI apparaissent particulièrement pesants ; l'importance des configurations locales d'acteurs sur la structuration des mises en œuvre territoriales.

Des

décisions

départementales à portée relative
L'analyse des décisions départementales laisse apparaître des formes de changement assez diversifiées : alors que la décision elle-même semble restreinte du fait des héritages de la décentralisation du RMI, les critères techniques de fonctionnement du dispositif contiennent des modalités d'inflexion importantes.

La mise en œuvre de l'expérimentation du RSA, dispositif optionnel, relève avant tout d'un choix politique des Présidents de Conseils généraux: elle vise à valoriser la capacité de choix du Président du Conseil général et à introduire des éléments de récit ponctuant l'histoire des territoires (Le Bart, 1990 ; Radaelli, 2000).

À l'origine de la décision d'expérimenter, les acteurs interrogés mentionnent à tous coups une rencontre entre le Président du Conseil général et Martin Hirsch, Haut commissaire aux solidarités actives. Ils soulignent toujours l'adéquation qui se fait jour entre les préoccupations de leur Président et celles du Haut Commissaire. Cette décision apparaît d'autant plus importante qu'elle concerne un processus d'expérimentation. L'enjeu est fort ici de démontrer le système d'attribution causale qui préside à cette décision (Le Bart, 1990) et ainsi de mettre en valeur la capacité d'affirmation du Président.

Du point de vue de la décision d'expérimenter, le changement peut apparaître important. Néanmoins, dès les premiers échanges, il est largement fait mention des héritages du RMI et du poids de la gestion de ce dispositif sur la prise de décision. 
Les acteurs départementaux sont partagés lorsqu'ils mentionnent l'influence de la décentralisation du RMI sur leurs manières d'appréhender l'expérimentation du RSA. Cette expérience leur sert à la fois pour démontrer leur expertise et la continuité dans laquelle ils se placent pour expérimenter mais aussi pour souligner les difficultés, notamment, dans leurs relations avec l'État sur ce dossier.

Du point de vue de l'évolution du système de protection sociale, le changement le plus net apparaît bien davantage à l'occasion de la décentralisation du RMI qu'à l'occasion de l'expérimentation du RSA. Ici, la dimension temporelle du changement joue pleinement: l'expérimentation s'ancre dans chaque département dans la continuité des réformes élaborées en matière de territorialisation des politiques sociales, de politiques départementales d'insertion ou encore d'accompagnement des bénéficiaires du RMI. Ces choix pèsent fortement sur les techniques d'appropriation de l'expérimentation (Pierson, 2000).

Par conséquent, les initiatives prises par les Départements avant l'expérimentation jouent largement sur la façon d'intégrer et de s'approprier celle-ci. Plus les formes choisies s'approchent du modèle préconisé dans le cadre de l'expérimentation, plus cette dernière est aisée.

Les acteurs soulignent eux-mêmes l'interdépendance entre les phases de décentralisation puis d'expérimentation pour expliquer les crispations politiques autour du RMl et les inquiétudes qui en découlent pour la mise en œuvre du RSA :

" Le débat sur le RSA a quand même été un peu écrasé par un autre débat ou, en tout cas, par le contexte : le coût du RMI et surtout la charge que cela fait peser sur les Départements, du fait de la noncompensation intégrale. Ici, c'est évident, les élus étaient quand même beaucoup plus préoccupés par cette question, parce qu'ici c'est massif, les chiffres sont importants et ça a masqué finalement d'autres débats qui auraient pu avoir lieu, y compris des débats très politiques sur le RSA : l'emploi, la structure de l'emploi, le développement de l'emploi précaire, etc. » (Un responsable du Conseil général du Nord).

Bien que la phase d'expérimentation ait été l'occasion de tester un certain nombre de pistes et de réponses nouvelles aussi bien en terme de montage institutionnel que d'accompagnement des personnes, l'impression qui domine localement est bien celle d'une expérimentation inachevée et précipitée. Pourtant, les changements introduits sont assez sensibles lorsque l'on s'intéresse aux instruments de l'expérimentation. 


\section{Le changement dans la continuité}

\section{a. Des critères d'accès, instruments politiques de la décision}

En période d'expérimentation, les contours des critères d'accès à la prestation, établis dans les Départements, sont révélateurs, non seulement de l'appropriation du dispositif par les acteurs locaux, mais également du contenu politique qu'ils leur donnent (Lorrain, 2004). Cet aspect permet de comprendre la diversité des situations envisagées par les assemblées départementales ainsi que la capacité à proposer des réponses nouvelles, variées, tenant compte des partenariats locaux et des situations des bénéficiaires (Fabre et Sautory, 2009). Au-delà des aspects techniques et de suivi des personnes, cette hétérogénéité des situations renvoie à des questionnements sur les normes et les valeurs départementales attachées à la question de l'insertion et de la lutte contre les exclusions mais aussi aux inégalités territoriales d'accès aux droits des personnes.

Deux critères semblent particulièrement révélateurs de cette situation :

- La population cible de l'expérimentation

Chaque département a dû définir le champ des allocataires du RMI qui pourront bénéficier du RSA expérimental. On distingue, le "stock » de bénéficiaires, c'est-à-dire les personnes déjà en emploi au moment du démarrage des expérimentations et le " flux » de bénéficiaires reprenant un emploi après la date de démarrage des expérimentations. Les Départements ne sont pas toujours explicites sur les raisons de leur choix vis-à-vis du "stock» et du "flux » des bénéficiaires. Néanmoins, ce choix révèle un plus ou moins grand souci de maîtrise des dépenses liées à ce nouveau dispositif.

Au sein des départements étudiés, trois territoires prennent en compte à la fois le "stock " et le "flux " de bénéficiaires (la Côte-d'Or, la LoireAtlantique et le Val-d'Oise). Dans ces départements, les entretiens montrent une grande adhésion au dispositif. Ceci peut aussi s'expliquer par un nombre assez réduit de personnes identifiées comme potentiellement bénéficiaires en Côte d'Or et Loire-Atlantique et par conséquent par une certaine générosité dans l'expérimentation du dispositif.

L'Eure et le Nord ont choisi, quant à eux, de ne prendre en compte que le " flux ». Dans le cas du Nord, le nombre important de bénéficiaires du RMI ainsi que les incertitudes quant au financement du dispositif RSA sont clairement mis en avant pour justifier la prise en compte des seuls « flux » de bénéficiaires.

La marge de manœuvre des départements concernant la définition de la population cible de l'expérimentation apparaît donc fortement conditionnée par le poids des dépenses sociales dans chaque territoire. L'accroissement des dépenses liées aux transferts de compétences a, de fait, participé à réduire l'autonomie financière des collectivités locales (Le Galès, 2006). 
- Les barèmes de calcul du RSA

Les barèmes adoptés apparaissent révélateurs de la volonté politique de chacun des départements. En effet, dans la majorité des départements, la formule de calcul choisie ${ }^{4}$ correspond à celle qui a été appliquée par l'État aux allocataires de l'Allocation parent isolé (API) également concernés par l'expérimentation du RSA. Sur ce point, les Conseils généraux ont d'ailleurs justifié l'utilisation de ce barème par un souci de simplicité.

Seuls la Loire-Atlantique et l'Eure ont fait jouer leur marge de manœuvre. En Loire-Atlantique, les conséquences du RSA, formulées en termes d'injustice sociale par rapport aux travailleurs au SMIC et aux travailleurs pauvres, ont justifié un barème un peu moins favorable. A l'inverse, dans l'Eure, l'objectif est d'atteindre au plus vite le seuil de pauvreté : le calcul est plus généreux que dans les autres départements. L'idée de départ est d'avoir un système " sur mesure " répondant au mieux aux besoins des personnes. Lors de la première vague d'enquête, les acteurs se félicitent grandement de l'originalité du dispositif dans leur département. Le calcul proposé leur semble particulièrement adapté pour répondre aux besoins des personnes. Cet enthousiasme ira en déclinant au fil de l'expérimentation tant le calcul proposé est difficile à instruire et ralentit la procédure d'instruction des dossiers.

Une autre dimension intéressante, qui éclaire la portée de la décision politique départementale réside dans le montage partenarial du RSA, notamment par rapport aux pratiques liées au RMI. Dans ce cas aussi, le changement, sans introduire de rupture fondamentale, est notable.

\section{b. L'ouverture des partenariats locaux : différents modes de coopération}

Que les politiques sociales reposent sur des partenariats ouverts à de nombreux acteurs publics et associatifs, n'est incontestablement pas une chose nouvelle (Geddes, 2000) ; Le Galès et Loncle, 2001). Néanmoins, les partenariats mobilisés dans le cadre de l'expérimentation du RSA se révèlent à la fois classiques et élargis. Ils sont classiques lorsque l'on considère les institutions et les organismes concernées : Conseil général, Caisse d'allocations Familiales/Mutualité sociale agricole, Préfecture, Direction départementale du travail, de l'emploi et de la formation professionnelle, Plans locaux pour l'insertion et l'emploi, Maisons de l'emploi, associations intermédiaires...

Ils sont élargis lorsque l'on s'intéresse au rôle joué par certains de ces acteurs: c'est le cas dans le cadre du suivi des bénéficiaires de l'Allocation de parent isolé pour les CAF et la Préfecture ; c'est également le cas du rôle imparti aux bénéficiaires dans le cadre du suivi des bénéficiaires du RMI par le Conseil général. De ce point de vue, le RSA entraîne des changements importants dans le suivi des personnes et dans l'intégration institutionnelle du dispositif. Bien sûr, d'un cercle

${ }^{4}$ RSA = RMI taux plein - min (allocation logement/forfait logement) - autres ressources $-0,3 \times$ revenus du travail. 
d'acteurs à un autre, les partenariats ne sont pas de même nature et n'entraînent pas les mêmes degrés de coopération.

S'agissant des CAF, leur rôle, déjà fortement consolidé par la décentralisation du RMI (Avenel, 2008), se trouve renforcé. En effet, au delà de la politique de contrôle plus systématique inaugurée depuis 2004 (Avenel, Donné et Sautory, 2008), certaines CAF sont conduites ici à renouveler leurs démarches d'accompagnement des bénéficiaires de l'API. De ce point de vue, les agents rencontrés soulignent à quel point l'introduction de l'expérimentation du RSA redonne du sens à leur métier. Une autre innovation partenariale se trouve dans le rôle joué par les publics cibles. Même si cette mobilisation a connu des sorts divers en fonction des départements, - elle apparaît ainsi difficile dans le Nord, éphémère dans l'Eure, ambitieuse en Loire-Atlantique- elle introduit des changements de pratiques intéressants dans la mise en œuvre des politiques publiques.

Ainsi, de l'aveu des professionnels des services centraux, c'était souvent la première fois qu'ils rencontraient des bénéficiaires de minima sociaux, et a fortiori la première fois qu'ils débattaient avec eux de leurs besoins, de leurs avis sur les prestations proposées. Ceci conduit à des évolutions des perceptions des usagers mais aussi des manières de procéder.

Les bénéficiaires participant aux groupes témoins émettent des avis positifs sur cette implication :

«Globalement, c'était enrichissant parce que cela permettait de s'exprimer sur quelque chose qui n'existait pas encore et on avait l'impression qu'on pouvait éventuellement changer quelque chose "...

«Au fil des comptes-rendus, on s'est aperçu que l'expression qu'on donnait était prise en compte. Ça conforte dans l'investissement » (Un bénéficiaire ayant participé à un groupe témoin).

Ainsi, l'ouverture des partenariats à de nouveaux types d'acteurs a-t-elle permis, en phase d'expérimentation, une meilleure connaissance des profils et des besoins des bénéficiaires du RSA ainsi qu'une meilleure adéquation des actions proposées, notamment par les CAF.

Le diagnostic partagé de l'échec des politiques d'insertion et le consensus des acteurs locaux autour de la nécessité de repenser les outils ont ainsi permis l'existence de changements dans la continuité pourtant instituée par l'héritage de la décentralisation (Streeck et Thelen, 2005 ; Palier, 2005).

Le poids de l'expertise : une impulsion nationale forte de l'ANSA

Bien que consacrant une marge de manœuvre non négligeable aux Départements, l'expérimentation du RSA repose sur des partenariats locaux qui sont notablement élargis, par rapport au RMI, à la demande de l'État et avec l'appui méthodologique de l'Agence nouvelle des solidarités actives. De ce point de vue, le RSA apparaît comme un dispositif à la fois décentralisé, certes, mais aussi administré à distance par l'État. Ce qui fait, notamment, l'originalité du processus c'est la place jouée par l'ANSA 
qui conseille, guide les départements en vue d'une cohérence d'ensemble à l'échelle nationale. Ce constat conduit néanmoins à des questionnements sur l'autonomie de la décision départementale.

On peut ainsi s'interroger sur l'intégration forte de l'ANSA dans le jeu institutionnel. En effet, cette agence, qui montre une volonté certaine de se distinguer de l'État, l'incarne tout de même en introduisant de nouveaux types de relations entre les instances de décision centrales et les collectivités locales ${ }^{5}$. Les acteurs départementaux, qu'ils relèvent des Conseils généraux, de la Préfecture ou de la CAF soulignent en effet combien l'ANSA joue un rôle primordial de conseil, d'expertise mais aussi de circulation d'informations et de pratiques entre les différents départements.

On peut voir dans cette dimension des éléments de changement radicalement nouveaux, soulignés par les acteurs locaux :

"On a dû faire remonter un certain nombre de situations, de problèmes: à quel moment se fait la rupture dans le statut de l'allocataire, à quel moment est-ce plus intéressant d'activer les droits ou de clôturer les droits? On a eu des débats sur toutes ces décisions, on a eu un jeu de questions-réponses, de validation ou d'annulation, qui a été opéré entre l'ANSA, la chargée de projet RSA, les techniciens du PLEPS [service territorialisé du Conseil général] et les référents généralistes de parcours du territoire. II y a vraiment eu un travail d'échange qui s'est opéré entre tous ces gens » (un référent généraliste de parcours, Département du Nord).

De fait, l'ANSA intervient à toutes les étapes de la prise de décision et aide même au montage opérationnel :

« L'ANSA était en situation idéale, avec un support politique au plus haut niveau, un président qui fait confiance et qui ne varie pas tous les matins. (...) On était en situation d'assistance très opérationnelle; nous avons participé au recrutement des référents RSA : on était là lors des premiers entretiens entre les référents et les bénéficiaires » (représentant de l'ANSA dans l'Eure).

À ce titre, l'ANSA apparaît comme promotrice du RSA dans les territoires et comme membre du partenariat constitutif de l'expérimentation au même titre que le Conseil général ou la CAF. Cette présence de l'ANSA aux côtés des acteurs clefs de l'expérimentation est révélatrice d'un changement profond dans les manières d'organiser les relations entre l'État et les collectivités locales. Ainsi, l'État n'apparaît plus comme une

${ }^{5}$ De ce point de vue, elle rejoint les descriptions des agences proposées par Daniel Benamouzig et Julien Besançon (2008) : "Elles sont les relais des politiques publiques dans des secteurs définis ou auprès de populations spécifiques. Elles sont généralement conçues de façon conjoncturelle, comme une solution de rechange à un recours à l'administration centrale. Personnes morales distinctes de l'État, les agences restent soumises à sa tutelle, au contrôle de la légalité et de la régularité des actes publics. Mais elles échappent à la relation hiérarchique de l'administration » (p. 285). 
force descendante et dotée de pouvoirs de contrainte vis-à-vis des territoires mais comme une instance régulatrice, présente dans les territoires et pourvoyeuse de conseils et d'expertise. On voit bien les mérites de cette nouvelle manière d'administrer: l'Agence est une toute petite entité, elle est peu consommatrice en personnels et en ressources pourtant elle apparaît indispensable aux Conseils généraux qui y trouvent un appui crucial $^{6}$. Dans le même temps, les conseils de l'Agence permettent de lisser les réponses départementales et les innovations proposées.

Par conséquent, au regard de l'ampleur du changement introduit et sur les capacités d'autonomie décisionnelle des départements, le changement apparaît ici contraint d'une double manière: par les héritages du RMI, qui impriment leurs marques sur les décisions prises par les départements dans leurs modes d'expérimentation; par l'ANSA qui, en préconisant des solutions nationales à des réponses locales, réduit la marge d'innovation des départements. La décision d'expérimenter apparaît ainsi de faible portée. Néanmoins, des changements indéniables existent: les types d'acteurs concernés et les règles techniques d'administration de la prestation évoluent. On peut ainsi considérer que le changement impulsé, même relatif, est réel même si c'est dans la technique que l'on trouve toute la mesure des positionnements politiques des décideurs locaux.

Des mises en œuvre inégalement innovantes
Si l'on accorde une attention particulière au changement au stade de la mise en œuvre de l'expérimentation du RSA, c'est parce que c'est à ce moment de l'implantation du dispositif que les décalages entre l'impulsion nationale et les réalités locales se font sentir le plus fortement (Padioleau, 1982 ; Kübler, 2000).

Dans notre analyse, ces décalages sont essentiellement le fait des configurations locales d'acteurs: ils trouvent leur expression dans les valeurs des agents, dans leurs marges de manœuvre par rapport à leur hiérarchie (Lipsky, 1980), dans les types de relations établies entre membres d'institutions différentes mais présents localement (Herjn et Porter, 1981).

L'impulsion nationale et la décision départementale, malgré leur importance, peuvent ainsi rencontrer des formes d'érosion non négligeables sur le terrain (Hudson et Lowe, 2009). À l'inverse, les acteurs locaux font parfois preuve de capacités d'innovation non négligeables fondées sur leur connaissance antérieure de la question traitée, sur la qualité des travaux partenariaux déjà engagés, sur le charisme ou la légitimité de tel ou tel acteur local (Loncle, 2009).

\footnotetext{
${ }^{6}$ Ce type de processus a déjà été largement observé pour le niveau européen (Majone, 1997 ; Dehousse, 1997) et se diffuse dans l'espace national dans les politiques sociales sous l'effet de phénomènes d'harmonisation cognitive (Palier, 2000) et de contrainte par les idées (Guigner, 2009).
} 
Dans le cas de l'expérimentation du RSA, les configurations locales peuvent être qualifiées de la manière suivante dans leur rapport au changement :

Tableau 1 - Configurations locales d'acteurs et modalités d'intégration du changement

\begin{tabular}{|c|c|c|}
\hline $\begin{array}{c}\text { Configuration locale } \\
\text { amplificatrice }\end{array}$ & $\begin{array}{c}\text { Configuration locale } \\
\text { adaptative }\end{array}$ & $\begin{array}{c}\text { Configuration locale } \\
\text { réductrice }\end{array}$ \\
\hline $\begin{array}{c}\text { Combinaison de } \\
\text { facteurs internes et } \\
\text { externes }\end{array}$ & $\begin{array}{c}\text { Facteurs externes } \\
\text { dominants }\end{array}$ & $\begin{array}{c}\text { Facteurs internes } \\
\text { dominants }\end{array}$ \\
\hline $\begin{array}{c}\text { Eure et Loire- } \\
\text { Atlantique }\end{array}$ & $\begin{array}{c}\text { Côte d'Or et Val } \\
\text { d'Oise }\end{array}$ & Nord \\
\hline
\end{tabular}

Insistons, pour le cas du département du Nord, sur le fait que la résistance au changement est spécifique au cas de l'expérimentation du RSA. Nos études antérieures sur la jeunesse, le sida, la prestation spécifique dépendance puis l'APA ou bien encore le RMI ont permis de montrer que ce territoire était plutôt porteur d'innovations. Ici, la résistance est d'ordre politique (opposition à l'État, notamment sur le dossier du RMI) et cognitif (incertitudes sur le fait que les bénéficiaires soient réellement gagnants dans le passage au RSA).

Les décalages sont également tangibles du fait des plus ou moins grandes capacités à influer sur les dynamiques d'insertion dans les territoires. Ceci est vrai lorsque l'on compare, par exemple, les situations des territoires de Nantes (Loire-Atlantique) et de Maubeuge (Nord). L'un est de petite taille, urbain et situé dans un département à fort dynamisme économique et dans la ville centre de ce même département; l'autre est étendu, à dominante rurale, dans un département qui peine face à la crise économique et fort éloigné de la ville centre. L'influence des acteurs publics et associatifs apparaît nettement plus facile à exercer dans le premier territoire que dans le second.

De même, la question de l'adéquation entre les qualifications des personnes et les attentes des chefs d'entreprise trouve des formes de résolution plus ou moins aisée dans les territoires en fonction des structures de l'emploi local. Ainsi, à Beaune (Côte-d'Or) et dans le territoire d'Argenteuil/Beuzons (Val d'Oise), les demandes du marché du travail apparaissent assez homogènes et rendent les modalités de réponse, sinon plus aisées, au moins plus lisibles que dans des territoires où les attentes du marché sont extrêmement floues et fluctuantes, comme à Maubeuge.

Enfin, les décalages de la mise en œuvre peuvent être amplifiés par des difficultés qui dépassent le cadre du territoire d'expérimentation. Cette dimension est observée au cours de la deuxième vague d'enquête : les acteurs étaient inquiets par rapport aux évolutions touchant l'ANPE et les ASSEDIC, ils étaient mécontents vis-à-vis du raccourcissement de la durée prévue de l'expérimentation, ils rencontraient également de 
nombreuses incertitudes du fait de la crise économique qui tendait à toucher fortement le secteur de l'intérim et de l'emploi peu qualifié.

Du point de vue du changement, l'analyse de la mise en œuvre apparaît donc féconde puisqu'elle permet de prendre la mesure des initiatives effectivement entreprises, de leur portée mais également de leurs limites, que ces dernières soient dues à des facteurs internes au territoire d'implantation ou à des éléments externes venant contraindre l'action publique locale.

\section{Les innovations institutionnelles dues à l'expérimentation}

Les motifs d'engagement dans le RSA peuvent varier selon les Conseils généraux, mais la conduite de l'expérimentation est présentée par les départements comme une opportunité pour interroger la manière de mettre en œuvre les politiques d'insertion, notamment en matière d'accompagnement des bénéficiaires. À des degrés divers, l'expérimentation est l'occasion de repenser ces politiques et donne lieu, le cas échéant, à un essai de réorganisation des dispositifs d'insertion. Dans la lignée des observations incrémentalistes (Lindblom, 1959) et néoinstitutionnalistes (Pierson, 2000), les capacités d'innovations institutionnelles dont sont dotés les agents des territoires étudiés apparaissent importantes ainsi que les dimensions cognitives qui marquent les orientations du changement au niveau local (Sabatier, 1986 ; Hall, 1993, 2000).

Une attention particulière portée au rôle des acteurs et à leurs logiques d'action permet de penser le changement malgré les obstacles institutionnels (Streeck et Theleen, 2005). Ainsi, dans le contexte d'une remise en cause des fondements cognitifs des politiques d'insertion par la récurrence de leurs échecs depuis la fin des années 1980 (Hall, 1993, 2000), l'adhésion des divers acteurs locaux aux orientations de l'expérimentation, à la redéfinition du problème de l'insertion, à ses principes de légitimation, influe fortement sur les capacités d'innovation des territoires considérés. Là encore, l'entrée par les configurations locales d'acteurs est féconde pour comprendre ce qui se joue dans les échanges ainsi que la portée du changement en cours.

Sur la question des innovations institutionnelles liées à l'expérimentation, certains départements se situent au stade de la réflexion, d'autres sont allés plus loin dans la mise en œuvre d'un nouveau fonctionnement des services, une révision des dispositifs d'insertion ou bien encore une nouvelle orientation en matière de coopération entre acteurs et institutions. En tout état de cause, un des acquis de l'expérimentation systématiquement mentionné est d'avoir suscité une (re)mobilisation des acteurs sur la question de l'insertion, et de porter un regard nouveau sur les dispositifs ainsi que sur les stratégies à mettre en œuvre.

Des éléments convergents se dégagent, tels que la mise en place de plateformes d'accompagnement permettant d'intégrer la question de l'accompagnement vers et dans l'emploi à travers l'association de partenaires comme l'ANPE, les maisons de l'emploi ou encore les entreprises d'insertion. 
S'agissant de la Loire-Atlantique, l'exemple de la Cellule RSA est tout à fait éclairant sur les impulsions qui ont pu être données à l'occasion de l'expérimentation. Cette cellule a pour objectif de renforcer les parcours pour que les allocataires RSA accèdent à un emploi qui les rende autonomes. Elle regroupe les différents professionnels de l'emploi (agent d'insertion du Conseil Général, agent ANPE, agent de la Maison de l'emploi ou conseillère emploi du CCAS) et du social (assistantes sociales du Conseil général, référents CAF); les entretiens proposés aux bénéficiaires du RSA sont conduits simultanément par un référent social et un référent emploi. Comparativement à un accompagnement classique la décision d'accompagnement est prise collectivement. La présence de tous les acteurs de l'insertion permet de désigner le référent le plus adapté à la situation professionnelle de l'allocataire, grâce aux compétences mises en commun.

Ce type d'innovation illustre les capacités d'adaptation des acteurs grâce à l'introduction du RSA. Celui-ci fait fonction d'une opportunité pour apporter du changement à la fois institutionnel et cognitif : la cellule RSA, instance partenariale permettant de travailler de concert sur les questions sociales et professionnelles.

Cette réponse est très ancrée dans les interactions propres aux acteurs composant la configuration locale: elle repose sur des dynamiques spécifiques et des valeurs propres à cet espace d'expérimentation et renvoie aux problèmes particuliers de ce territoire. Pour bien comprendre cette dernière dimension, on peut s'intéresser aux territoires d'expérimentation proposés par les Conseils généraux.

\section{Choix des territoires d'expérimentation et impact sur la mise en œuvre}

La question territoriale apparaît centrale dans l'expérimentation du RSA : en demandant aux Conseils généraux de choisir eux-mêmes l'espace de mise en œuvre du dispositif, le Haut commissaire et l'ANSA élargissent les possibles pour les acteurs départementaux.

De ce fait, le choix des territoires donne lieu à des débats (en Assemblée départementale ou au sein des territoires) : la couleur politique des élus territoriaux, les frontières géographiques du territoire quand celui-ci ne correspond pas à un échelon administratif prédéterminé, la structure économique, les types de qualification des bénéficiaires... sont autant d'éléments de détermination du territoire.

Celui-ci est donc ici envisagé dans une dimension alliant déconcentration et territorialisation : il est perçu comme un espace-test de mise en œuvre permettant de tirer des enseignements sur l'implantation locale d'un dispositif national et comme un espace actif, réagissant de manière singulière à l'introduction de ce dispositif - comment ce territoire particulier et les acteurs des différents domaines sont-ils en capacité de s'approprier la mesure et de proposer des modalités d'adaptation singulières ? (Le Galès, 1999 ; Tallard, Théret et Uri, 2002). 
Si l'on synthétise les démarches suivies, il apparaît que le choix final fait suite à une démarche de concertation entre les acteurs du Conseil général, de la Préfecture, de l'ANSA et du territoire pressenti, en suivant deux types de raisonnements : leur représentativité ou leur exemplarité.

Certains Départements ont choisi leur territoire pour leur représentativité : c'est le cas de la Côte-d'Or et du Nord qui ont sélectionné des territoires équilibrés, correspondant à une moyenne départementale. Le raisonnement qui préside dans ce cas est de mesurer la portée de l'expérimentation dans la perspective de la généralisation: les innovations, les enseignements tirés pourront servir pour l'ensemble du département.

Les autres Départements ont choisi leur territoire pour leur exemplarité. II s'agit d'exemplarité " positive » pour l'Eure et le Val-d'Oise qui optent pour un territoire dynamique sur le plan économique. II s'agit d'exemplarité «négative» pour la Loire-Atlantique où le territoire d'expérimentation est marqué par de nombreuses difficultés sociales. Dans les deux cas, des pistes singulières, des innovations spécifiques seront testées pour vérifier la capacité de changement propre au dispositif mais ces adaptations devront faire l'objet de révision en vue de la généralisation.

En cours de mise en œuvre, l'influence du choix du territoire sur la perception de l'expérimentation par les décideurs est fondamentale. Alors que certains territoires sont apparus facilitateurs (c'est le cas du territoire du CCAS de Nantes qui est apparu aisé à mobiliser du fait de sa petite taille et de son caractère homogène), d'autres se sont révélés plus problématiques (c'est le cas de l'Avesnois où le peu d'offres d'emploi pèse de manière négative sur le fonctionnement du dispositif). II ne semble pas que le RSA soit susceptible d'améliorer beaucoup une situation locale dominée par l'absence d'offres d'emploi, par l'isolement des acteurs de l'accompagnement et par l'éloignement vis-à-vis de la ville-centre. Dans ce cas de figure, même si la dynamique de partenariat local apparaît riche et potentiellement en capacité de produire des innovations, elle n'est cependant pas suffisante pour répondre à l'ampleur des questions d'insertion des bénéficiaires du territoire.

On peut donc se demander plus largement quelle est la part du changement potentiellement apporté par le RSA. Ce dernier semble, en effet, en mesure de susciter des innovations institutionnelles intéressantes du point de vue de l'accompagnement des bénéficiaires mais il paraît aussi fondamentalement limité lorsqu'il s'agit d'influer sur les situations locales de l'emploi. Ce constat est d'autant plus problématique lorsqu'il est mis en perspective avec les difficultés rencontrées par les acteurs locaux au moment de la mise en œuvre. 


\section{Les difficultés de mise en œuvre comme freins au changement}

Dans l'étude de la mise en œuvre de l'expérimentation du RSA, il apparaît important de relater les difficultés auxquelles ont à faire face les acteurs décisionnels et de terrain: elles permettent de saisir les mécanismes internes et externes qui introduisent des décalages entre les formulations nationales, les décisions départementales et la réalité de terrain. Trois éléments ont retenu notre attention.

a. Les difficultés d'harmonisation des pratiques et de connaissance des publics

L'analyse de l'expérimentation laisse émerger un sentiment mitigé quant à l'innovation permise par l'évolution des modalités d'accompagnement. Ainsi, si l'on constate de la part des Conseils généraux et des CAF des tentatives pour proposer de nouvelles pratiques (rendez-vous à échéances plus rapprochées avec les bénéficiaires, horaires d'ouverture élargis, meilleure connaissance de la structure familiale de l'ayant droit...), des difficultés concrètes restent fortes (de grandes disparités dans les profils des référents, des informations peu fournies sur le RSA auprès des professionnels, des insuffisances de moyens - par exemple, un accès à internet très limité pour certains professionnels - ), de même que les difficultés d'harmonisation.

A ce propos, l'exemple du département du Nord est illustratif s'agissant du passage des dossiers des bénéficiaires de l'API au RMI (quand l'enfant des bénéficiaires atteint l'âge de trois ans). Ainsi que le soulignent les professionnels de la CAF, les acteurs ne savent pas encore très bien comment procéder au moment du changement de statut de la personne.

Les questions, qui touchent la CAF, le Conseil général et l'ANSA, se posent en effet de la manière suivante :

" On a peu, pour le moment, de passages RSA-API à RSA-RMI mais, quand le premier s'est présenté, j'ai demandé : "voilà, on a une dame qui bascule du RSA-API en RSA-RMI, comment procède-t-on ?" Donc la question a été transmise à la responsable qui supervise la cellule RSA... et voilà. Ça remonte à juin et je n'ai pas de nouvelles à ce jour. Donc on en a rediscuté au comité de pilotage RSA-API qui a eu lieu il y a 15 jours et l'ANSA nous proposait finalement d'inviter le Conseil général au prochain comité de pilotage pour qu'on puisse clairement débattre notamment de cette situation... il pourrait y avoir d'autres choses à discuter " (Un membre de la CAF).

Ces difficultés sont ressenties par les bénéficiaires qui soulignent combien les institutions peuvent être "déconnectées " et ne pas se représenter totalement les réelles conditions d'existence et les contraintes rencontrées par les bénéficiaires de minima sociaux. Au-delà de ces difficultés qui peuvent être considérées comme internes à la mise en œuvre du RSA, un des points d'achoppement du dispositif est la faible participation des entreprises. 


\section{b. Les tentatives inabouties d'ouverture au monde du travail}

La mise en œuvre du RSA suppose la mobilisation et la collaboration avec les acteurs de l'emploi présents dans le territoire.

Au titre des acteurs publics, le Conseil général et la CAF travaillent avec l'ANPE, les Plans locaux d'insertion par l'économique (PLIE), les entreprises d'insertion par l'économique, les Maisons de l'emploi, éventuellement les plateformes de services pour la création d'entreprises... Bien sûr, là encore, en fonction des caractéristiques du territoire d'expérimentation et des habitudes de partenariat, les relations sont plus ou moins étroites et fluides.

Dans les Départements, ces relations sont généralement établies à partir du Plan départemental d'insertion (PDI) ou autre document qui a vocation à élargir la politique d'insertion. Cependant, au-delà des documents officiels, l'existence des PDI n'est pas toujours très concluante (Vald'Oise) ou bien tend à masquer des différences de fonctionnement entre acteurs du même type (entre les deux PLIE présents dans le territoire d'expérimentation dans le Nord). Néanmoins, les Départements ont, par le biais du RSA, renforcé leurs attentes pour une inscription des allocataires du RMI auprès des services de l'ANPE.

Avec les entreprises elles-mêmes, les relations sont les plus compliquées. Si l'on excepte les tentatives menées par les Conseils généraux de l'Eure et du Val d'Oise, on peut dire que l'expérimentation du RSA n'a eu qu'une portée moyenne sur le partenariat avec les entreprises. Même dans ces départements, au-delà de la volonté institutionnelle affichée par le Conseil général, les résultats ne sont pas encore très importants. Les entretiens menés avec les entreprises laissent apparaître une grande indifférence. Seuls les groupements d'employeurs comme le Mouvement des entreprises de France (MEDEF) ou les Chambres de commerce et d'industrie $(\mathrm{CCl})$ ou bien encore les fédérations d'employeurs à domicile semblent trouver un intérêt au RSA. Pour le reste, les entreprises ont majoritairement refusé les entretiens ou alors ont fait part de leur peu d'intérêt pour le RSA.

\section{c. Les incertitudes liées au contexte de l'expérimentation}

Les acteurs rencontrés estiment être placés dans une période d'incertitude qui nuit à l'investissement plein et entier dans ce processus.

À l'issue de la deuxième vague d'enquête, à l'automne 2008, les acteurs de quatre des cinq départements se disent très insatisfaits de la façon dont l'expérimentation a été conduite (en particulier, au regard des raccourcissements des délais de l'expérimentation initialement prévus et du manque de recul qui en découle). Les acteurs ont largement exprimé leurs doutes sur la manière dont le RSA généralisé pourra être financé, sur la capacité réelle de l'État à suivre l'implantation dans les départements, sur les implications de la réforme ANPE- ASSEDIC, sur les risques induits par le "profilage » de deux filières d'accompagnement (une professionnelle, une sociale). 
L'expérimentation semble avoir rendu les acteurs locaux globalement circonspects vis-à-vis du dispositif à la fois du fait du contexte institutionnel et politique général qu'ils vivent comme défavorable aux départements et du fait des spécificités du RSA.

Conclusion
On peut insister sur le caractère relatif du changement introduit par l'expérimentation du RSA.

Du point de vue de la décision, la portée du changement est limitée : l'introduction de l'expérimentation du RSA se fait dans une grande continuité avec la décentralisation du RMI, d'une part, et dans une concertation forte avec l'ANSA, d'autre part. Les marges de manœuvre qui sont laissées aux décideurs et qui sont investies comme autant d'éléments politiques portent sur des questions techniques - pente de calcul, public bénéficiaire, droits connexes - qui seront harmonisées au moment de la généralisation. Dans cette perspective, l'autonomie des départements vis-à-vis de l'impulsion du changement apparaît relative.

Du point de vue de la mise en œuvre, les innovations institutionnelles se heurtent à la réalité de l'implantation territoriale qui peut se révéler très épineuse malgré les impulsions politiques. Si l'on ne peut pas nier l'existence d'une ambition au changement de la part du Haut commissariat et de la part des décideurs départementaux, cette ambition doit faire face à de nombreuses limites : le changement a bien lieu mais il est d'une ampleur plus restreinte que ce qui a été souhaité par les décideurs et il est avant tout placé dans la continuité des réformes de décentralisation.

En reprenant les précautions méthodologiques introductives et en mobilisant une analyse du changement ancrée dans la temporalité, ouverte aux différentes phases d'ancrage de l'action publique et prenant en compte les différents ordres de changement, on peut spécifier la nature du changement en cours (Hall, 1993). On peut ainsi affirmer que le changement est indéniable du point de vue des instruments et des acteurs mobilisés - le RSA introduit une réforme d'ampleur dans la prise en charge des bénéficiaires des minima sociaux : les Conseils généraux, CAF et ANSA, triangle porteur de l'expérimentation. En revanche, le changement est plus limité si l'on considère son cadre d'interaction et ses orientations politiques.

Sur ces deux dimensions, on observe de nombreuses difficultés en termes de décision autant que de mise en œuvre: les positionnements réciproques des acteurs restent flous à bien des égards, certains sont absents malgré leur importance (les entreprises), les cadres cognitifs sont essentiellement le résultat de l'héritage de la décentralisation du RMI.

Ces ampleurs différenciées peuvent être expliquées par la nature des leviers à la disposition des Départements : alors que ces derniers peuvent convaincre les agents de leurs services ainsi que leurs partenaires à faire évoluer leurs modalités d'action et de collaboration, ils peinent à faire 
entrer des acteurs qui ne tombent pas sous leur influence, ici les entreprises. C'est dans ce domaine que les capacités de changement d'un dispositif pourtant innovant comme le RSA semblent les plus fragiles.

\section{BIBLIOGRAPHIE}

AVENEL C. (2008), "Trois ans après la décentralisation du RMI. L'analyse du rôle des caisses d'allocations familiales " dans Minima sociaux. Diversité des logiques d'action et des publics, Recherches et Prévisions, CNAF, $n^{\circ}$ 91, mars, p. 57-68.

AVENEL C., DONNE S., SAUTORY O. (2008), « L'organisation du RMI dans le cadre de la décentralisation ", in Lelièvre M., Nauze-Fichet E. (eds), RMI, l'état des lieux, Paris, La Découverte, p. 213-226.

BENAMOUZIG D., BESANÇON J. (2008), « Les agences: de nouvelles administrations publiques? " in Borraz O., Guiraudon V. (eds), Les politiques publiques en France, vol 1, La France dans la gouvernance européenne, Paris, Presses de Sciences po, p. 283-307.

BORRAZ O. (1998), Gouverner une ville, Besançon, 1959-1989, Rennes, Presses universitaires de Rennes, 1998.

BORRAZ O., LONCLE P. (2000), "Permanences et recompositions du secteur sanitaire, les politiques locales de lutte contre le sida ", Revue française de sociologie, vol 41, $\mathrm{n}^{\circ} 1$, p. 37-60.

CYTERMANN L., DINDAR C. (2008) : « Les grandes étapes de l'histoire du RMI ", in Lelièvre M., Nauze-Fichet E. (eds), RMI, l'état des lieux, Paris, La Découverte, p. 23-50.

DEHOUSSE R. (1997), "Regulation by Network in the European Community: the Role of European Agencies », Journal of European Public Policy, vol. 4, $\mathrm{n}^{\circ}$ 2, p. 246-261.

DESTREMEAU B., MESSU M. (2008) « Le droit à l'assistance sociale à l'épreuve du local ", Revue française de science politique, vol $58, \mathrm{n}^{\circ}$ 5, p. 713-740.

FABRE V., SAUTORY O. (2009), « Enquête sur les expérimentations du RSA : premiers résultats ", Document de travail, DREES, $\mathrm{n}^{\circ} 87$, avril, $18 p$.

GEDDES M. (2000), "Tackling social exclusion in the European Union ? The limits of the new orthodoxy of local partnership", International journal for urban and regional research, vol. 24, p. 782-800.

GREMION P. (1976), Le Pouvoir périphérique: bureaucrates et notables dans le système politique français, Paris, Seuil.

GUIGNER S. (2009), « L'Union européenne, acteur contemporain de la biopolitique : l'exemple de la lutte contre le tabagisme ", intervention au colloque de l'Association française de science politique, Grenoble.

HALL P. (1993), "Policy Paradigm, Social Learning and the State ", Comparative Politics, vol. 25, $\mathrm{n}^{\circ} 3$, p. 275-296.

HALL P. (2000) «Le rôle des intérêts, des institutions et des idées dans l'économie politique comparée des pays industrialisés", Revue internationale de politique comparée, $n^{\circ} 7,2000$, p. 53-92. 
HEJRN B., PORTER D. (1981), « Implementation Structures: a New Unit of Administrative Analysis », Organization Studies, 2, p. 211-227.

HUDSON J., LOWE S. (2009) Understanding the policy process, analysing welfare policy and practice, Bristol, Policy Press, (second edition).

KÜBLER D. (2000), Politique de la drogue dans les villes suisses entre ordre et santé, analyse des conflits de mise en œuvre, Paris, L'Harmattan.

LASCOUMES P., LE BOURHIS J.P (1998), " Le Bien commun comme construit territorial. Identités d'action et procédures », Politix, vol. 11, $\mathrm{n}^{\circ} 42$, p. 37-66.

LE BART C. (1990), "Le système des attributions causales dans le discours des candidats à l'élection présidentielle de 1988 », Revue française de science politique, vol 40, $\mathrm{n}^{\circ} 2$, p. 212-229.

LE BIHAN B., MARTIN C., RIVARD T. (2006), « L'organisation du RMI et de son volet insertion dans neuf départements depuis la décentralisation », DREES, Etudes et Résultats, n²35.

LE GALES P. (1999), "Régulation, gouvernance et territoire », in Commaille J., Jobert $\mathrm{B}$. (eds), Les métamorphoses de la régulation politique, Paris, LGDJ, p. 203-240.

LE GALES P. (2006), «Les deux moteurs de la décentralisation. Concurrences politiques et restructuration de l'Etat jacobin », dans $P$. Culpepper, P. Hall et B. Pallier (dir.), La France en mutation. 19802005, Paris, Presses de Sciences Po., p. 303-341.

LE GALÈS P., LONCLE P. (2001), "Local partnerships and social inclusion in France: experiences and ambiguities", in Geddes M., Benington J. (eds), Local partnerships and social inclusion in European Union, London, Routledge, p. 70-91.

LE LIDEC P. (2008), « La réforme des institutions locales » in Borraz O., Guiraudon V. (eds), Politiques publiques, 1. La France dans la gouvernance européenne, Paris, Presses de sciences po, p. 255-281.

LINDBLOM C. (1959), "The Science of Muddling Through", Public Administration Review, vol. 19, p. 79-88.

LIPSKY M. (1980), Street-Level Bureaucracy: Dilemmas of the Individual in Public Services, New York, Russel Sage Fondation.

LONCLE P. (2009), « La mise en œuvre des politiques de santé publique au niveau local: "l'animation territoriale en santé en Bretagne" », Sciences sociales et santé, vol $27, \mathrm{n}^{\circ} 1$, p. 5-31.

LONCLE P., MUNIGLIA V., RIVARD T., ROTHE C. (2008), « Fonds d'aide aux jeunes et inégalités territoriales : aide a minima ou politiques départementales de jeunesse?", Revue française des affaires sociales, $n^{\circ} 1$, p. 229-249.

LORRAIN D. (2004), «Les pilotes invisibles de l'action publique, le désarroi du politique? », in Lascoumes P., Le Galès P. (eds), Gouverner par les instruments, Paris, Presses de Sciences po, p. 163-197. 
MAJONE G. (1997), «The New European Agencies: regulation by information », Journal of European Public Policy, vol 4, n² 2, p. 262275.

NEGRIER E. (2005), La question métropolitaine, les politiques à l'épreuve du changement d'échelle territoriale, Grenoble, Presses universitaires de Grenoble.

PADIOLEAU J.-G. (1982), L'État au concret, Paris, Presses universitaires de France.

PALIER B. (2000), « Does Europe matter? Européanisation et réformes des politiques sociales des pays de l'Union européenne, Politique européenne, $n^{\circ} 2$, p. 7-28.

PALIER B. (2005), "Ambiguous Agreement, Cumulative Change : French Social Policy in France", in Streek W., Thelen K. (eds), Beyond Continuity, Institutional Change in Advanced Political Economies, Oxford, Oxford University Press, p. 127-144.

PASQUIER R. (2004), La capacité politique des régions, une comparaison France/Espagne, Rennes, Presses universitaires de Rennes.

PIERSON P. (2000), « Path Dependence, Increasing Returns, and the Study of Politics », American Political Sicence Review, 94 (2), p. 251267.

RADAELLI C. (2000), "Logiques de pouvoir et récits dans l'Union européenne ", Revue française de science politique, vol $50, n^{\circ} 2, p$. 255-276.

SABATIER P. (1986), "Top-Down and Bottom-up approaches to implementation research: a critical analysis and suggested synthesis ", Journal of public policy, $n^{\circ} 6$, p. 21-48.

STREECK W., THELEN K. (2005), "Introduction: Institutional Change in Advanced Political Economies", in W. Streeck et K. Thelen (eds.), Beyond Continuity. Institutional Change in Advanced Political Economies, Oxford, Oxford UP, p. 1-39.

TALLARD M., THERET B., URI D. (eds) (2002), Innovations institutionnelles et territoires, Paris, L'Harmattan.

THIERRY M. (2008), « La décentralisation du RMI : responsabiliser les départements, mais pas seulement les départements ", in Lelièvre M., Nauze-Fichet E. (eds), RMI, l'état des lieux, Paris, La Découverte, p. 243-249.

WARIN P., AVENEL C. (2007), «Les Conseils généraux dans la décentralisation du RMI », Pouvoirs locaux, n²5, p. 54-61. 